\title{
Solid-liquid equilibrium in the system 2-keto-L-gulonic acid + sodium-2-keto-L-gulonate + water
}

\author{
Fabian Jirasek $^{\mathrm{a}}$, Jakob Burger ${ }^{\mathrm{b}, *}$, Hans Hasse $^{\mathrm{a}}$ \\ ${ }^{a}$ Laboratory of Engineering Thermodynamics (LTD), University of Kaiserslautern, Erwin-Schrödinger-Str. 44, \\ 67663 Kaiserslautern, Germany \\ ${ }^{b}$ Chair of Chemical Process Engineering, Technical University of Munich, Campus Straubing for Biotechnology \\ and Sustainability, Schulgasse 16, 94315 Straubing, Germany
}

\begin{abstract}
The solid-liquid equilibrium (SLE) in the ternary system 2-keto-L-gulonic acid (HKGA) + sodium2-keto-L-gulonate (NaKGA) + water was studied experimentally at temperatures between 275 and $313 \mathrm{~K}$ and ambient pressure. At these conditions, HKGA and NaKGA precipitate as monohydrates: HKGA $\cdot \mathrm{H}_{2} \mathrm{O}$ and $\mathrm{NaKGA} \cdot \mathrm{H}_{2} \mathrm{O}$, respectively. Phase diagrams with one eutonic point are found for all temperatures. A thermodynamic model of the SLE that is based on an extended version of the Debye-Hückel theory was developed and the dissociation constant of HKGA as well as the solubility products of $\mathrm{HKGA} \cdot \mathrm{H}_{2} \mathrm{O}$ and NaKGA $\cdot \mathrm{H}_{2} \mathrm{O}$ were determined. The agreement between the experimental data and the results from the model is excellent.
\end{abstract}

Keywords: solid-liquid equilibrium, 2-keto-L-gulonic acid, thermodynamic model, Debye-Hückel

\section{Introduction}

2-keto-L-gulonic acid (HKGA) is produced on a large scale as an intermediate in the L-ascorbic acid (vitamin $\mathrm{C}$ ) process. While for a long time HKGA was produced via the mainly chemical route proposed by Reichstein and Güssner [1], fermentation became the dominant production process

5 after novel bioconversion processes had been developed $[2,3,4,5,6]$. The isolation and purification of products from fermentation broths is challenging because of the complexity and the high water content of the broths. Therefore, downstream processing in biotechnological processes usually includes several separation steps. HKGA precipitates as monohydrate $\mathrm{HKGA} \cdot \mathrm{H}_{2} \mathrm{O}$ from acidic solutions at ambient pressure $[7,8]$. However, during fermentation, the $\mathrm{pH}$ has to be adjusted to neutral conditions. This is usually done by adding basic sodium-containing electrolytes like sodium carbonate $\left(\mathrm{Na}_{2} \mathrm{CO}_{3}\right)[9,10]$ or sodium hydroxide $(\mathrm{NaOH})[8,11,12]$. In the presence of sodium ions at neutral conditions, the sodium salt of HKGA sodium-2-keto-L-gulonate (NaKGA) can precipitate from aqueous solutions. Thereby, also NaKGA precipitates as monohydrate NaKGA . $\mathrm{H}_{2} \mathrm{O}$ at ambient pressure $[7,13]$. Literature on the solubilities of HKGA and NaKGA in water

\footnotetext{
*Corresponding author

Email address: burger@tum.de (Jakob Burger)
} 
is scarce. Domschke et al. [14] report the solubility of HKGA in water to be $0.5 \mathrm{~g} / \mathrm{g}$ at $323 \mathrm{~K}$. According to Dümpelmann et al. [7], the solubilities of pure HKGA and pure NaKGA in water at $303 \mathrm{~K}$ are $480 \mathrm{~g} / \mathrm{l}$ and $250 \mathrm{~g} / \mathrm{l}$, respectively. Systematic SLE data of the ternary system HKGA + NaKGA + water is not available in the literature. This work therefore provides comprehensive experimental SLE data of the system HKGA + NaKGA + water in the industrially relevant temperature range (275-313 K) at ambient pressure. Furthermore, the dissociation constant of HKGA and the solubility products of HKGA $\cdot \mathrm{H}_{2} \mathrm{O}$ and NaKGA $\cdot \mathrm{H}_{2} \mathrm{O}$ were determined and a thermodynamic model of the SLE in the studied system is presented, which is based on an extended version of the Debye-Hückel theory [15].

\section{Materials and Methods}

\subsection{Chemicals}

Anhydrous 2-keto-L-gulonic acid (HKGA) was obtained from Cargill. Sodium hydroxide (NaOH) pellets were purchased from Sigma-Aldrich. For all experiments, ultra pure water produced with a Milli-Q machine (Elix Essential 5, Millipore) was used. Table 1 gives an overview of all chemicals that were used in the present work. It includes the chemicals for the preparation of the eluents for the ion chromatographic analysis.

Table 1: Suppliers and purities of chemicals used in this work.

\begin{tabular}{l|l|l|l}
\hline \hline Chemical name (abbreviation) & Formula & Supplier & Purity (mass fraction) \\
\hline 2-keto-L-gulonic acid (HKGA) & $\mathrm{C}_{6} \mathrm{H}_{10} \mathrm{O}_{7}$ & Cargill & $>0.98$ \\
Sodium hydroxide & $\mathrm{NaOH}$ & Sigma-Aldrich & $>0.99$ \\
Sodium carbonate & $\mathrm{Na}_{2} \mathrm{CO}_{3}$ & Th. Geyer & $>0.998$ \\
Sodium hydrogen carbonate & $\mathrm{NaHCO}_{3}$ & Sigma-Aldrich & $>0.997$ \\
Nitric acid 0.1N & $\mathrm{HNO}_{3}$ & Merck & $>0.99$ \\
\hline \hline
\end{tabular}

\subsection{Experimental Procedure}

Mixtures of HKGA, $\mathrm{NaOH}$, and water were prepared as follows using a laboratory scale (AG204, Mettler Toledo). Suspensions were prepared by pouring about $5 \mathrm{~g}$ of water into $30 \mathrm{ml}$ glass vessels and adding about $5 \mathrm{~g}$ of anhydrous HKGA while stirring with a magnetic stirrer. Different amounts of an aqueous solution of $0.5 \mathrm{~g} / \mathrm{g} \mathrm{NaOH}$ (prepared from $\mathrm{NaOH}$ pellets and water) were added to the vessels. The amount of $\mathrm{NaOH}$ was varied in a way to achieve samples with molar ratios of $\mathrm{NaOH}$ to HKGA ranging from zero to one. For obtaining neutral mixtures, the HKGA was completely neutralized by adding the stoichiometric amount of $\mathrm{NaOH}$. After the preparation of the mixtures, a solid phase was observed in all vessels. The vessels were sealed and equilibrated 40 at constant temperature in an incubator (ICP600, Memmert) for 5 days. The solid phase was 
kept suspended during the equilibration by magnetic stirring. The temperature was measured with a calibrated PT100 thermometer connected to a digital multimeter (5017, Prema) with an accuracy of $\pm 0.1 \mathrm{~K}$. After the equilibration, the stirring was stopped to allow the solid phase to settle. Samples were drawn from the clear supernatant using syringes with syringe filters $(5 \mu \mathrm{m}$ 45 pore size, Rotilabo, Carl Roth), which had been thermostated previously at the temperature of the experiment. The samples were gravimetrically diluted with water using the laboratory scale and analyzed by ion chromatography (930 Compact IC Flex system, cation exchange column Metrosep C 6-250/4.0 with $4.0 \mathrm{mmol} / \mathrm{l}$ nitric acid as eluent, anion exchange column Metrosep A Supp 5-250/4.0 with an aqueous solution of $3.2 \mathrm{mmol} / \mathrm{l}$ sodium carbonate and $1.0 \mathrm{mmol} / \mathrm{l}$ sodium hydrogen carbonate as eluent, Metrohm). In the analysis, the concentrations of sodium ions $\left(\mathrm{Na}^{+}\right)$ and 2-keto-L-gulonate ions $\left(\mathrm{KGA}^{-}\right)$were determined. The uncertainty of the mass fractions of the ions was below $2 \%$, as demonstrated by analyzing synthetic samples of known composition. To elucidate the stoichiometry of the precipitated species, solid phase was separated from the liquid by filtration, dried in a drying chamber at ambient pressure and $323 \mathrm{~K}$, dissolved in water, and analyzed as described before. Under the assumption that both crystal species HKGA and NaKGA are only present as monohydrates according to the literature (cf. $[7,8,13])$, three cases were distinguished: if no $\mathrm{Na}^{+}$is found, the solid is only $\mathrm{HKGA} \cdot \mathrm{H}_{2} \mathrm{O}$; if the same amount of $\mathrm{Na}^{+}$ and $\mathrm{KGA}^{-}$is found, the solid is only NaKGA $\cdot \mathrm{H}_{2} \mathrm{O}$; if more $\mathrm{KGA}^{-}$than $\mathrm{Na}^{+}$is found, the solid contains both species. The concentration of hydrate water in the solid phase was calculated from mass balances confirming the exclusive presence of the monohydrate forms of both solids. In preliminary experiments, it was confirmed that an equilibration time of 5 days is sufficient to reach the solid-liquid equilibrium in the investigated system at the studied conditions. For the determination of the dissociation constant of HKGA, a liquid sample of HKGA and water with a defined concentration was prepared gravimetrically. The $\mathrm{pH}$ of this sample was measured at different temperatures with a $\mathrm{pH}$ meter (780, Metrohm).

\section{Model}

Figure 1 shows a schematic of the model of the solid-liquid equilibrium in the system HKGA + NaKGA + water. The model takes into account the dissociation of HKGA and NaKGA in the liquid phase as well as the autoprotolysis of water in the liquid phase. Since HKGA is a weak electrolyte, it is present in both the dissociated form $\left(\mathrm{H}^{+}\right.$and $\left.\mathrm{KGA}^{-}\right)$and the undissociated form (HKGA) in the liquid phase. NaKGA is a strong electrolyte and fully dissociated in the liquid phase. The solid can be either pure HKGA $\cdot \mathrm{H}_{2} \mathrm{O}$ or pure $\mathrm{NaKGA} \cdot \mathrm{H}_{2} \mathrm{O}$ or both. The equilibrium constant of the dissociation of HKGA is $K_{\mathrm{HKGA}}^{\text {diss }}$ and that of the autoprotolysis of water is $K_{\mathrm{H}_{2} \mathrm{O}}^{\text {diss }}$, respectively. The solid-liquid phase equilibrium is characterized by the solubility 
75 products $K_{\mathrm{HKGA} \cdot \mathrm{H}_{2} \mathrm{O}}^{\mathrm{SP}}$ and $K_{\mathrm{NaKGA} \cdot \mathrm{H}_{2} \mathrm{O}}^{\mathrm{SP}}$.

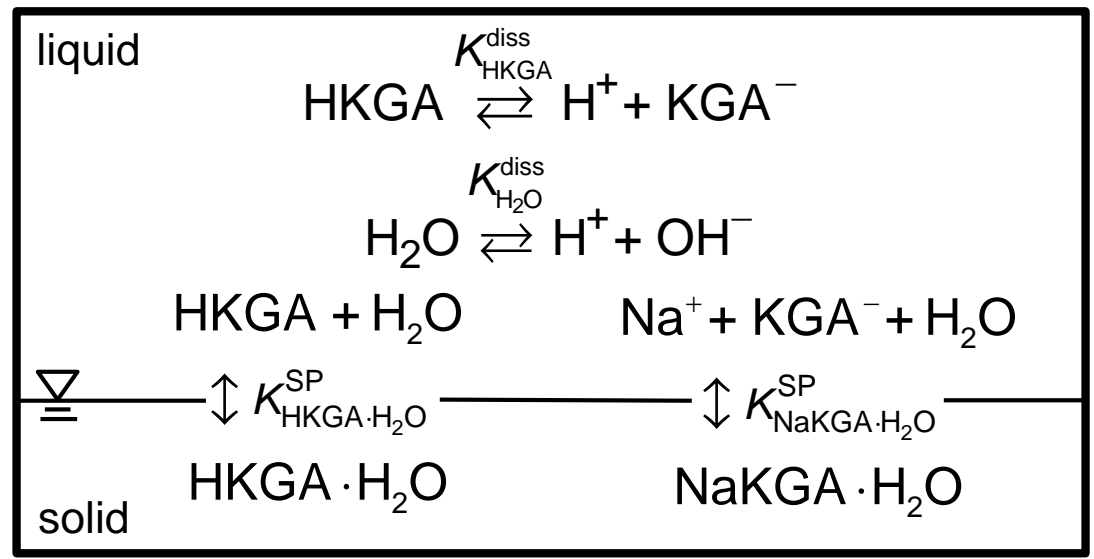

Figure 1: Schematic of the model of the solid-liquid equilibrium in the ternary system HKGA + NaKGA + water.

The thermodynamic equilibrium constants are defined by Eqs. 1 to 4 .

$$
\begin{aligned}
K_{\mathrm{HKGA}}^{\mathrm{diss}} & =\frac{m_{\mathrm{H}^{+}} \cdot m_{\mathrm{KGA}^{-}}}{m_{\mathrm{HKGA}} \cdot m_{0}} \cdot \frac{\gamma_{\mathrm{H}^{+}} \cdot \gamma_{\mathrm{KGA}^{-}}}{\gamma_{\mathrm{HKGA}}} \\
K_{\mathrm{H}_{2} \mathrm{O}}^{\mathrm{diss}} & =\frac{m_{\mathrm{H}^{+}} \cdot m_{\mathrm{OH}^{-}}}{m_{0}^{2}} \cdot \frac{\gamma_{\mathrm{H}^{+}} \cdot \gamma_{\mathrm{OH}^{-}}}{a_{\mathrm{H}_{2} \mathrm{O}}} \\
K_{\mathrm{HKGA} \cdot \mathrm{H}_{2} \mathrm{O}}^{\mathrm{SP}} & =\frac{m_{\mathrm{HKGA}}}{m_{0}} \cdot a_{\mathrm{H}_{2} \mathrm{O}} \cdot \gamma_{\mathrm{HKGA}} \\
K_{\mathrm{NaKGA} \cdot \mathrm{H}_{2} \mathrm{O}}^{\mathrm{SP}} & =\frac{m_{\mathrm{Na}^{+}} \cdot m_{\mathrm{KGA}^{-}}}{m_{0}^{2}} \cdot a_{\mathrm{H}_{2} \mathrm{O}} \cdot \gamma_{\mathrm{Na}^{+}} \cdot \gamma_{\mathrm{KGA}^{-}}
\end{aligned}
$$

The variables $m_{k}$ and $\gamma_{k}$ represent the molality and the activity coefficient of species $k$ in the liquid phase, respectively. $m_{0}$ is defined as $1 \mathrm{~mol} / \mathrm{kg}$. The variable $a_{\mathrm{H}_{2} \mathrm{O}}$ denotes the activity of water in the liquid phase, which is normalized according to Raoult's law, whereas the activity coefficients of all other components (the solutes) are normalized similar to Henry's law. The activity coefficients $\gamma_{i}$ of the ionic species $i$ in the liquid phase are calculated as a function of the ionic strength $I$ in the liquid phase using an extended version of the Debye-Hückel theory [15], which is given in Eqs. 5 to 7.

$$
\begin{aligned}
\ln \gamma_{i} & =-A^{\Phi}\left(\frac{\sqrt{I}}{1+b \sqrt{I}}+\frac{2}{b} \ln (1+b \sqrt{I})\right) \\
A^{\Phi} & =\frac{1}{3}\left(2 \pi N_{\mathrm{A}} \rho_{\mathrm{H}_{2} \mathrm{O}} m_{0}\right)^{\frac{1}{2}}\left(\frac{e^{2}}{4 \pi \varepsilon_{0} \varepsilon_{\mathrm{H}_{2} \mathrm{O}} k T}\right)^{\frac{3}{2}} \\
I & =\frac{1}{2} \sum_{i} \frac{m_{i}}{m_{0}} z_{i}^{2}
\end{aligned}
$$

The nomenclature of the symbols is as follows: $b$ Debye-Hückel parameter (set to 1.2 as sug- 
${ }_{85}$ gested by Pitzer [15]), $N_{\mathrm{A}}$ Avogadro's number, $\rho_{\mathrm{H}_{2} \mathrm{O}}$ specific density of water, $e$ absolute elementary charge, $\varepsilon_{0}$ vacuum permittivity, $\varepsilon_{\mathrm{H}_{2} \mathrm{O}}$ relative permittivity of water, $k$ Boltzmann's constant, $T$ absolute temperature, $z_{i}$ charge number of ion $i$. The activity coefficient of the neutral species HKGA is assumed to be unity and the activity of water is calculated using the Gibbs-Duhem equation.

90 The equilibrium constant $K_{\mathrm{H}_{2} \mathrm{O}}^{\text {diss }}$ of the autoprotolysis of water was taken from the literature [16]. The remaining equilibrium constants $\left(K_{\mathrm{HKGA}}^{\text {diss }}, K_{\mathrm{HKGA} \cdot \mathrm{H}_{2} \mathrm{O}}^{\mathrm{SP}}, K_{\mathrm{NaKGA} \cdot \mathrm{H}_{2} \mathrm{O}}^{\mathrm{SP}}\right)$ were fitted to experimental data of the present work: the values of $K_{\mathrm{HKGA}}^{\text {diss }}$ were determined from experimental data on the $\mathrm{pH}$ of an aqueous solution with a known concentration of pure HKGA at different temperatures (cf. Supplementary Material). The values of $K_{\mathrm{HKGA} \cdot \mathrm{H}_{2} \mathrm{O}}^{\mathrm{SP}}$ and $K_{\mathrm{NaKGA} \cdot \mathrm{H}_{2} \mathrm{O}}^{\mathrm{SP}}$ were determined from experimental data on the solubility of pure HKGA and pure NaKGA in water at different temperatures, respectively.

\section{Results and Discussion}

The experimental SLE data are presented in Table 2. The solubilities of pure HKGA and pure NaKGA in water are plotted in Figure 2 as a function of the temperature. They increase with increasing temperatures. The solubility of HKGA is higher than that of NaKGA and shows a higher temperature dependency.

The values determined for $K_{\mathrm{HKGA}}^{\text {diss }}, K_{\mathrm{HKGA} \cdot \mathrm{H}_{2} \mathrm{O}}^{\mathrm{SP}}$, and $K_{\mathrm{NaKGA} \cdot \mathrm{H}_{2} \mathrm{O}}^{\mathrm{SP}}$ are shown in Figure 3 in a logarithmic plot over the inverse temperature. Linear relations are found. Thus, the temperature dependency of every equilibrium constant $K_{j}$ is modeled using the van't Hoff equation assuming values for the enthalpy change $\Delta h_{j}$ and entropy change $\Delta s_{j}$ that are independent of the temperature, cf. Eq. 8.

$$
\ln K_{j}=-\frac{\Delta h_{j}}{R} \cdot \frac{1}{T}+\frac{\Delta s_{j}}{R}
$$

where $R$ is the universal gas constant. The results for the parameters $\Delta h_{j}$ and $\Delta s_{j}$ from the fit are given in Table 3. The resulting solubilities of pure HKGA and pure NaKGA in water calculated by the model are shown as lines in Figure 2. There is excellent agreement of calculated solubilities and the experimental data. A comparison with the solubilities from the literature that are reported in mass concentrations is not possible as no data on the density of the solutions is available. The deviation between the solubility of pure HKGA in water at $323 \mathrm{~K}$ reported by Domschke et al. [14] and the solubility calculated by an extrapolation of the present model to $323 \mathrm{~K}$ is smaller than $2 \%$. 
Table 2: Experimental SLE data of the ternary system HKGA + NaKGA + water at ambient pressure and the studied temperatures $T$. The solid phase is either HKGA monohydrate (A) or NaKGA monohydrate (B) or both (A $+\mathrm{B})$. The composition of the liquid phase in equilibrium is specified using mass fractions $w$ of the solutes HKGA and $\mathrm{NaKGA}^{a}$.

\begin{tabular}{|c|c|c|c|c|c|c|c|c|}
\hline $\begin{array}{l}w_{\text {HKGA }} \\
\mathrm{g} / \mathrm{g}\end{array}$ & $\begin{array}{l}w_{\mathrm{NaKGA}} \\
\mathrm{g} / \mathrm{g}\end{array}$ & $\begin{array}{l}\text { Solid } \\
\text { phase }\end{array}$ & $\begin{array}{l}w_{\mathrm{HKGA}} \\
\mathrm{g} / \mathrm{g}\end{array}$ & $\begin{array}{l}w_{\text {NaKGA }} \\
\mathrm{g} / \mathrm{g}\end{array}$ & $\begin{array}{l}\text { Solid } \\
\text { phase }\end{array}$ & $\begin{array}{l}w_{\text {HKGA }} \\
\mathrm{g} / \mathrm{g}\end{array}$ & $\begin{array}{l}w_{\text {NaKGA }} \\
\mathrm{g} / \mathrm{g}\end{array}$ & $\begin{array}{l}\text { Solid } \\
\text { phase }\end{array}$ \\
\hline \multicolumn{9}{|c|}{$\bar{T} T=275.2 \mathrm{~K}$} \\
\hline 0.000 & 0.175 & $\mathrm{~B}$ & 0.187 & 0.149 & $\mathrm{~B}$ & 0.226 & 0.067 & $\mathrm{~A}$ \\
\hline 0.000 & 0.174 & $\mathrm{~B}$ & 0.212 & 0.146 & $A+B$ & 0.240 & 0.002 & $\mathrm{~A}$ \\
\hline 0.000 & 0.174 & $\mathrm{~B}$ & 0.215 & 0.145 & $\mathrm{~A}+\mathrm{B}$ & 0.240 & 0.001 & $\mathrm{~A}$ \\
\hline 0.070 & 0.165 & B & 0.216 & 0.146 & $A+B$ & 0.241 & 0.000 & $\mathrm{~A}$ \\
\hline 0.132 & 0.156 & $\mathrm{~B}$ & 0.216 & 0.125 & A & & & \\
\hline \multicolumn{9}{|c|}{$T=283.2 \mathrm{~K}$} \\
\hline 0.000 & 0.186 & $\mathrm{~B}$ & 0.218 & 0.152 & $\mathrm{~B}$ & 0.241 & 0.123 & $\mathrm{~A}$ \\
\hline 0.003 & 0.186 & $\mathrm{~B}$ & 0.227 & 0.153 & $\mathrm{~A}+\mathrm{B}$ & 0.261 & 0.067 & $\mathrm{~A}$ \\
\hline 0.003 & 0.185 & $\mathrm{~B}$ & 0.240 & 0.150 & $A+B$ & 0.277 & 0.001 & $\mathrm{~A}$ \\
\hline 0.043 & 0.181 & $\mathrm{~B}$ & 0.246 & 0.141 & $\mathrm{~A}+\mathrm{B}$ & 0.279 & 0.000 & $\mathrm{~A}$ \\
\hline 0.116 & 0.169 & $\mathrm{~B}$ & 0.249 & 0.147 & $A+B$ & & & \\
\hline 0.215 & 0.153 & $\mathrm{~B}$ & 0.250 & 0.148 & $\mathrm{~A}+\mathrm{B}$ & & & \\
\hline \multicolumn{9}{|c|}{$T=293.2 \mathrm{~K}$} \\
\hline 0.000 & 0.207 & $\mathrm{~B}$ & 0.281 & 0.152 & $A+B$ & 0.288 & 0.155 & $A+B$ \\
\hline 0.076 & 0.191 & $\mathrm{~B}$ & 0.282 & 0.153 & $\mathrm{~A}+\mathrm{B}$ & 0.289 & 0.163 & $A+B$ \\
\hline 0.113 & 0.185 & $\mathrm{~B}$ & 0.284 & 0.152 & $A+B$ & 0.308 & 0.095 & $\mathrm{~A}$ \\
\hline 0.122 & 0.183 & $\mathrm{~B}$ & 0.286 & 0.153 & $\mathrm{~A}+\mathrm{B}$ & 0.333 & 0.000 & $\mathrm{~A}$ \\
\hline 0.267 & 0.159 & $\mathrm{~B}$ & 0.286 & 0.153 & $A+B$ & 0.334 & 0.001 & $\mathrm{~A}$ \\
\hline \multicolumn{9}{|c|}{$T=303.0 \mathrm{~K}$} \\
\hline 0.000 & 0.223 & $\mathrm{~B}$ & 0.273 & 0.169 & $\mathrm{~B}$ & 0.325 & 0.156 & $A+B$ \\
\hline 0.006 & 0.221 & B & 0.311 & 0.162 & B & 0.330 & 0.159 & $A+B$ \\
\hline 0.042 & 0.215 & B & 0.320 & 0.156 & $\mathrm{~A}+\mathrm{B}$ & 0.333 & 0.157 & $A+B$ \\
\hline 0.042 & 0.215 & B & 0.322 & 0.156 & $A+B$ & 0.353 & 0.075 & $\mathrm{~A}$ \\
\hline 0.098 & 0.204 & $\mathrm{~B}$ & 0.323 & 0.156 & $\mathrm{~A}+\mathrm{B}$ & 0.387 & 0.002 & $\mathrm{~A}$ \\
\hline 0.148 & 0.194 & $\mathrm{~B}$ & 0.324 & 0.155 & $A+B$ & 0.388 & 0.000 & $\mathrm{~A}$ \\
\hline 0.186 & 0.185 & $\mathrm{~B}$ & 0.324 & 0.157 & $\mathrm{~A}+\mathrm{B}$ & & & \\
\hline \multicolumn{9}{|c|}{$\bar{T}=312.8 \mathrm{~K}$} \\
\hline 0.000 & 0.241 & $\mathrm{~B}$ & 0.362 & 0.156 & $A+B$ & 0.395 & 0.061 & $\mathrm{~A}$ \\
\hline 0.117 & 0.216 & B & 0.365 & 0.157 & $\mathrm{~A}+\mathrm{B}$ & 0.437 & 0.000 & $\mathrm{~A}$ \\
\hline 0.351 & 0.152 & $A+B$ & 0.369 & 0.158 & $A+B$ & & & \\
\hline 0.360 & 0.156 & $A+B$ & 0.381 & 0.121 & $\mathrm{~A}$ & & & \\
\hline
\end{tabular}

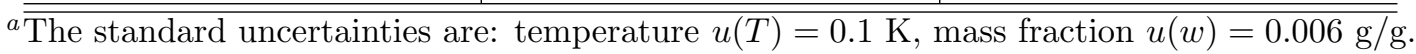




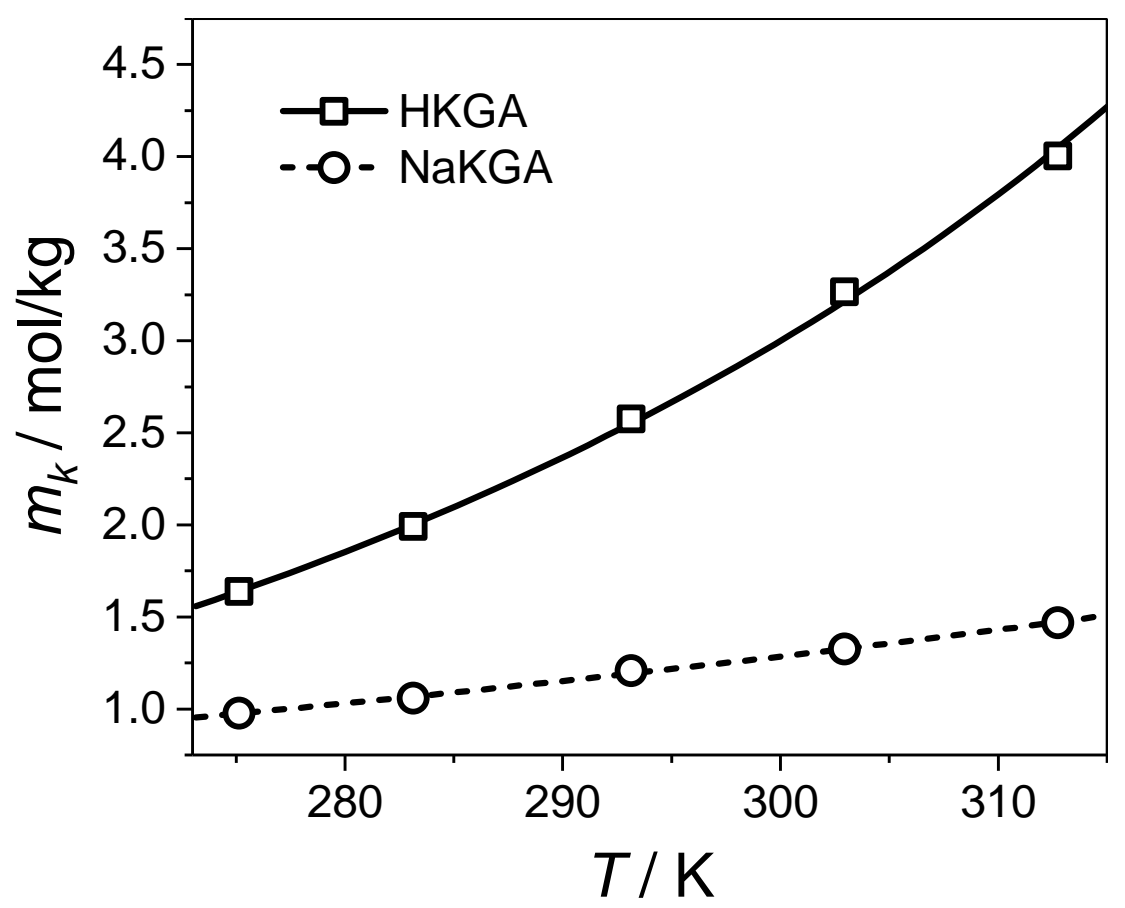

Figure 2: Solubilities of HKGA and NaKGA in water as a function of the temperature $T$. Symbols: experimental data (this work). Lines: model.

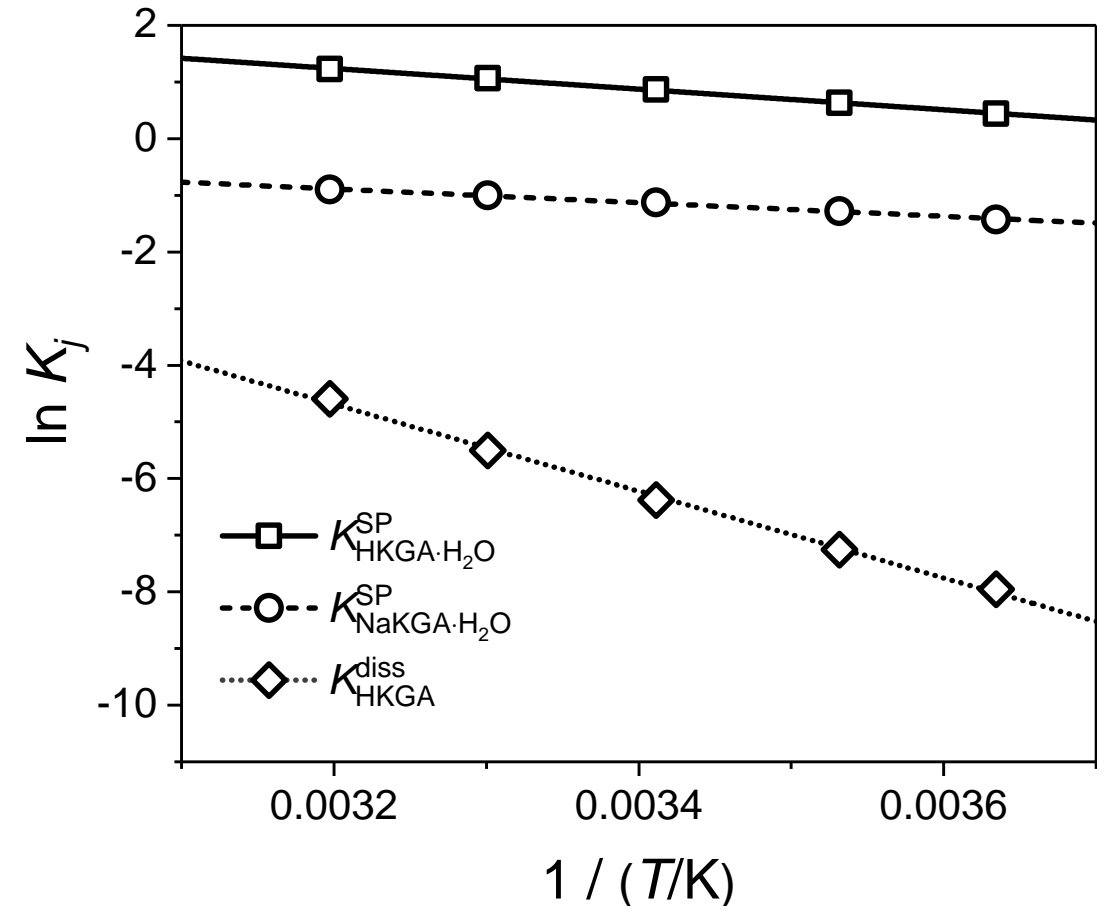

Figure 3: Equilibrium constants $K_{j}$ in a logarithmic plot over the inverse temperature $T$. Symbols: calculated from experiments of this work. Lines: model. 
Table 3: Parameters for the correlation of the temperature dependency of the equilibrium constants in Eq. 8.

\begin{tabular}{l|c|c|l}
\hline \hline Equilibrium constant & $\Delta h_{j} / \mathrm{kJ} / \mathrm{mol}$ & $\Delta s_{j} / \mathrm{J} /(\mathrm{mol} \cdot \mathrm{K})$ & Data for fit \\
\hline$K_{\mathrm{HKGS}}^{\mathrm{dis}}$ & 63.7 & 165.0 & This work \\
$K_{\mathrm{HKGAA} \cdot \mathrm{H}_{2} \mathrm{O}}^{\mathrm{SK}}$ & 15.1 & 58.7 & This work \\
$K_{\mathrm{NaKGA} \cdot \mathrm{H}_{2} \mathrm{O}}^{\mathrm{SP}}$ & 10.0 & 24.7 & This work \\
$K_{\mathrm{H}_{2} \mathrm{O}}^{\text {diss }}$ & 52.3 & -99.2 & Literature $^{a}$ \\
\hline \hline
\end{tabular}

a) Determined from data reported in [16]. for all investigated temperatures. Figure 4 shows the phase diagram for $303.0 \mathrm{~K}$. The diagram has four regions that meet in one eutonic point. Since both solid species crystallize as monohydrates, these regions are: L unsaturated solution, L+A saturated solution in equilibrium with HKGA monohydrate crystals, L+B saturated solution in equilibrium with NaKGA monohydrate crystals, $\mathrm{L}+\mathrm{A}+\mathrm{B}$ saturated solution in equilibrium with HKGA monohydrate crystals and NaKGA monohydrate crystals. Figure 4 contains experimental data that was obtained in the present work for the liquid phase composition in the SLE as well as results from the model. The agreement is almost perfect. Figure 5 gives an overview of the corresponding results for all temperatures that were studied in the present work. Again excellent agreement is observed.

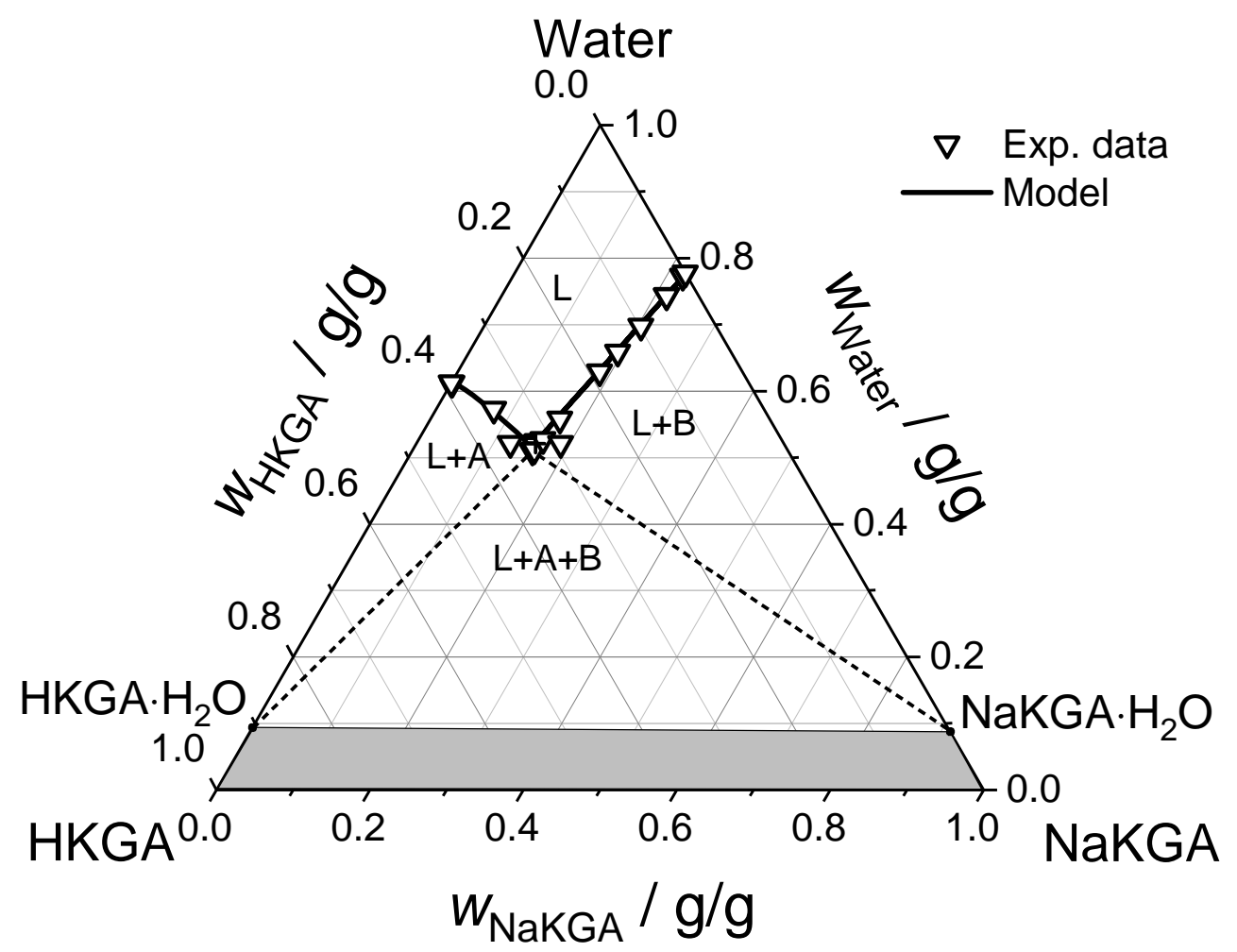

Figure 4: Phase diagram of the system HKGA + NaKGA + water at $303.0 \mathrm{~K}$ and ambient pressure. L: unsaturated solution. L+A: SLE, the solid phase is pure HKGA monohydrate (A). L+B: SLE, the solid phase is pure NaKGA monohydrate (B). L+A+B: SLE with two solid phases (A and B). Experimental data of the liquid phase composition in the SLE are represented by symbols. Solid line: model. In the shaded area, only solid phases coexist; this area was not studied here. 


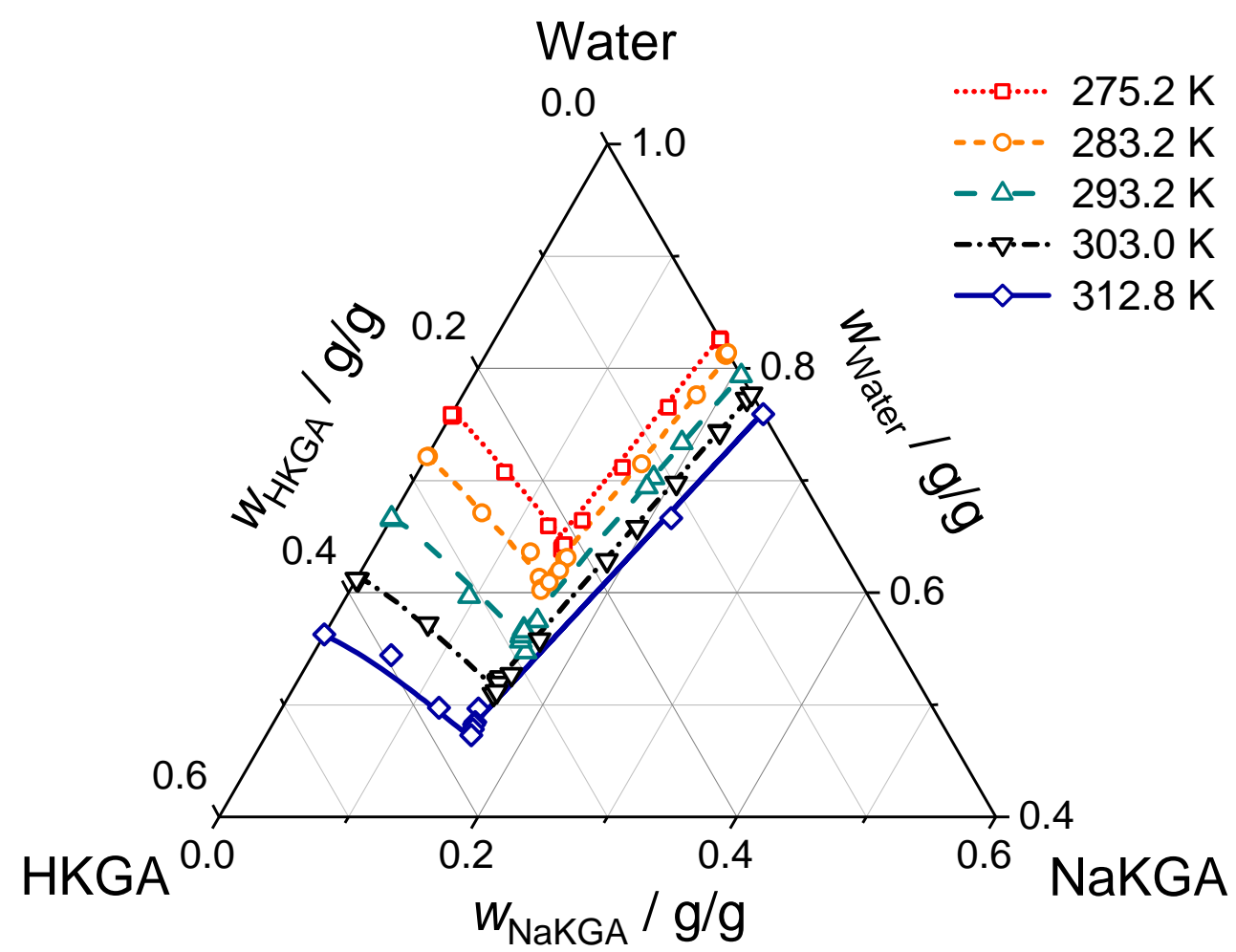

Figure 5: Composition of the liquid phase in SLE in the system HKGA + NaKGA + water at ambient pressure. The symbols (experimental data, this work) and lines (model) are in excellent agreement.

In Figure 6, the composition of the eutonic point in the system HKGA + NaKGA + water as calculated by the model is shown as a function of the temperature. With increasing temperature, the concentration of HKGA increases significantly whereas the concentration of NaKGA only slightly increases. These trends are similar to the trends found for the solubilities of pure HKGA and pure NaKGA in water (cf. Figure 2). 


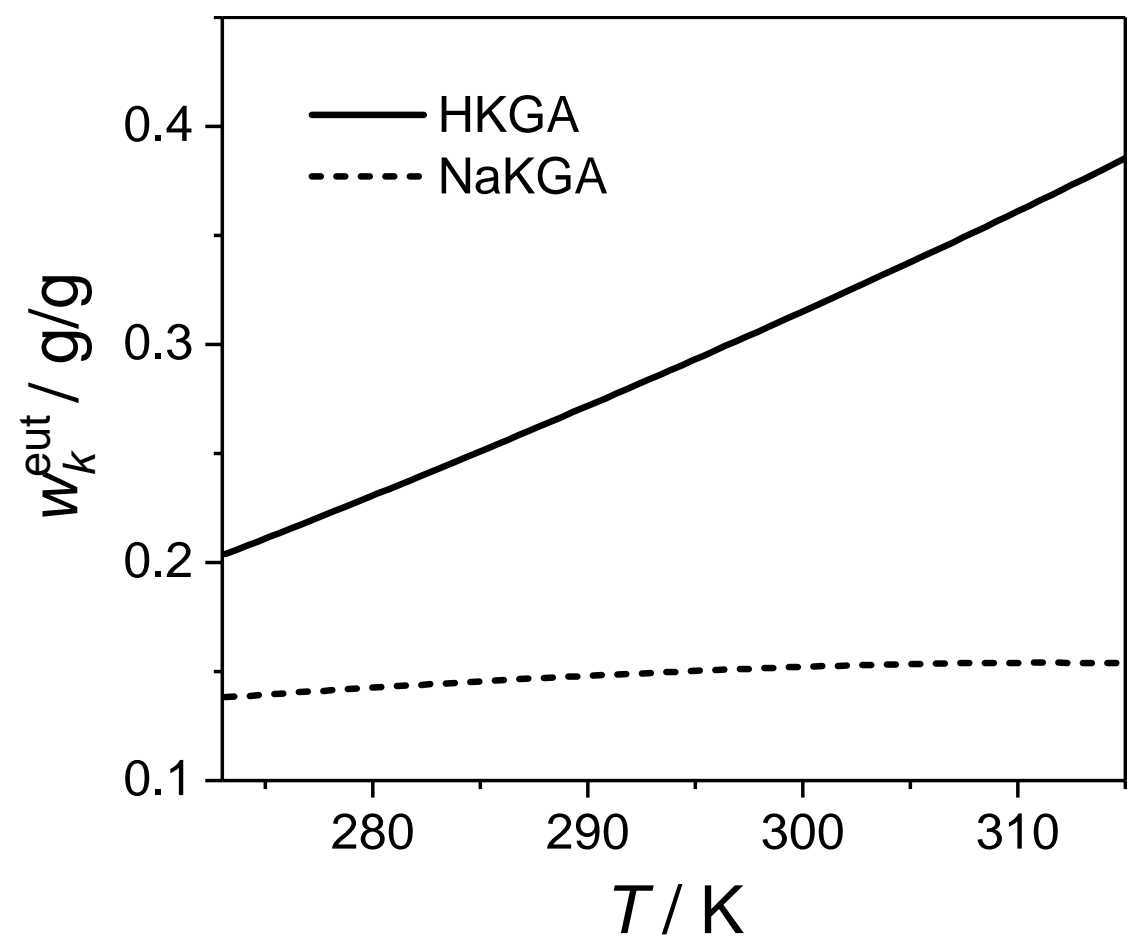

Figure 6: Mass fraction $w_{k}^{\text {eut }}$ of HKGA and NaKGA at the eutonic point in the system HKGA + NaKGA + water at ambient pressure as a function of the temperature $T$ calculated with the presented model.

In this work, a simple approach was used to model the activity coefficients (extended DebyeHückel theory and the assumption $\gamma_{\mathrm{HKGA}}=1$ ). The results show that this approach is sufficient to model the SLE in the studied system. The experimental data of this work do not allow a deeper validation of the activity coefficient model, as possible errors are veiled by the fit of the equilibrium constants.

A molality-based equilibrium model that does not require the calculation of activity coefficients is presented in the Supplementary Material. It yields a description of the experimental data that is only slightly worse than that from the activity-based model.

\section{Conclusion}

The downstream processing of fermentation broths containing 2-keto-L-gulonic acid (HKGA) 140 is an important step in the production of vitamin C. Crystallization is a favorable technique for separating HKGA from these solutions. During fermentation, the $\mathrm{pH}$ has to be adjusted to neutral conditions. Sodium-containing electrolytes like sodium hydroxide $(\mathrm{NaOH})$ are often used for this purpose. As a consequence, not only HKGA but also sodium-2-keto-L-gulonate (NaKGA) can precipitate. Hence, for process design and optimization, it is important to know the phase diagram of the system HKGA + NaKGA + water. That diagram was not available in the literature up to now. Therefore, in the present work, experimental data on the solid-liquid equilibrium in the system HKGA + NaKGA + water were taken at temperatures between 275 and $313 \mathrm{~K}$, i.e. 
in the range that is interesting for the production process. The phase diagrams show the same qualitative behavior with one eutonic point for all temperatures. HKGA and NaKGA do not precipitate as pure crystals but as monohydrates $\mathrm{HKGA} \cdot \mathrm{H}_{2} \mathrm{O}$ and $\mathrm{NaKGA} \cdot \mathrm{H}_{2} \mathrm{O}$, respectively. The solubility of HKGA in water is higher and more temperature-dependent than that of NaKGA. A physico-chemical model for the SLE in the system HKGA + NaKGA + water was developed. It takes into account the dissociations in the liquid phase as well as the physical non-ideality, which is described with an extension of the Debye-Hückel theory. The solubility products of HKGA $\cdot \mathrm{H}_{2} \mathrm{O}$ and $\mathrm{NaKGA} \cdot \mathrm{H}_{2} \mathrm{O}$ in water and the dissociation constant of HKGA in water were determined from the present data.

\section{Funding Sources}

This work was supported by the European Community's Framework Program for Research and Innovation Horizon 2020 (2014-2020) [grant agreement number 636077].

\section{Declarations of interest}

none 


\section{Nomenclature}

Symbols

$a \quad$ activity [-]

$b \quad$ Debye-Hückel parameter [-]

$e \quad$ absolute elementary charge $[\mathrm{C}]$

$h \quad$ molar enthalpy $[\mathrm{J} / \mathrm{mol}]$

$I \quad$ ionic strength $[\mathrm{mol} / \mathrm{kg}]$

$K \quad$ equilibrium constant [-]

$k \quad$ Boltzmann's constant $[\mathrm{J} / \mathrm{K}]$

$M \quad$ molar mass $[\mathrm{g} / \mathrm{mol}]$

$m$ molality $[\mathrm{mol} / \mathrm{kg}]$

$N_{\text {A }} \quad$ Avogadro's number $[1 / \mathrm{mol}]$

$R \quad$ universal gas constant $[\mathrm{J} /(\mathrm{mol} \cdot \mathrm{K})]$

$s \quad$ molar entropy $[\mathrm{J} /(\mathrm{mol} \cdot \mathrm{K})]$

$T \quad$ temperature $[\mathrm{K}]$

$w \quad$ mass fraction $[\mathrm{g} / \mathrm{g}]$

$z \quad$ relative charge number [-]

Greek letters

$\Delta \quad$ difference, change [-]

$\gamma \quad$ activity coefficient [-]

$\varepsilon_{0} \quad$ vacuum permittivity $[\mathrm{F} / \mathrm{m}]$

$\varepsilon_{\mathrm{H}_{2} \mathrm{O}}$ relative permittivity of water [-]

$\rho \quad$ specific density $\left[\mathrm{kg} / \mathrm{m}^{3}\right]$

Sub- and Superscripts

0 reference

Diss dissociation

eut eutonic point

$i \quad$ ionic species

j equilibrium reaction

$k \quad$ species

SP solubility product 
HKGA 2-keto-L-gulonic acid

$\mathrm{KGA}^{-} \quad$ 2-keto-L-gulonate ion

NaKGA sodium-2-keto-L-gulonate

SLE solid-liquid equilibrium

\section{References}

[1] T. Reichstein, A. Grüssner, Eine ergiebige Synthese der L-Ascorbinsäure (Vitamin C), Helvetica Chimica Acta 17 (1) (1934) 311-328. doi:10.1002/hlca.19340170136.

[2] C. S. Lopez-Garzon, A. J. Straathof, Recovery of carboxylic acids produced by fermentation, Biotechnology Advances 32 (5) (2014) 873 - 904. doi:10.1016/j.biotechadv.2014.04.002.

[3] J. Boudrant, Microbial processes for ascorbic acid biosynthesis: a review, Enzyme and Microbial Technology 12 (5) (1990) 322 - 329. doi:10.1016/0141-0229(90)90159-N.

[4] G. Pappenberger, H.-P. Hohmann, Biotechnology of Food and Feed Additives, Springer Berlin Heidelberg, Berlin, Heidelberg, 2014, Ch. Industrial Production of l-Ascorbic Acid (Vitamin C) and d-Isoascorbic Acid, pp. 143-188.

[5] C. Bremus, U. Herrmann, S. Bringer-Meyer, H. Sahm, The use of microorganisms in l-ascorbic acid production, Journal of Biotechnology 124 (1) (2006) 196205. doi:10.1016/j.jbiotec.2006.01.010.

[6] R. D. Hancock, R. Viola, Biotechnological approaches for l-ascorbic acid production, Trends in Biotechnology 20 (7) (2002) 299-305. doi:10.1016/s0167-7799(02)01991-1.

[7] R. Dümpelmann, T. Keglevic, Process for the conversion of the sodium salt of 2-keto-l-gulonic acid to the free acid, US 5852211 A (1998).

[8] J. De Troostembergh, I. Debonne, W. Obyn, C. Peuzet, Process for the manufacture of 2keto-l-gulonic acid, EP 1417324 A2 (2004).

[9] L. Yu, A. Lin, L. Zhang, C. Chen, W. Jiang, Application of electrodialysis to the production of vitamin c, Chemical Engineering Journal 78 (2) (2000) 153 - 157. doi:10.1016/S13858947(00)00136-4.

[10] W. Ning, Z. Tao, C. Wang, S. Wang, Z. Yan, G. Yin, Fermentation process for producing 2-keto-l-gulonic acid, EP 0278447 A2 (1988). 
[11] T. Hoshino, S. Ojima, T. Sugisawa, Process for producing 2-keto-l-gulonic acid, US 5312741 A (1994).

[12] K. Imai, T. Sakane, I. Nogami, Method for producing 2-keto-l-gulonic acid, US 4892823 A (1990).

[13] S. Dette, M. Sanselme, T. Stelzer, L. Renou, M. Jones, N. Couvrat, G. Coquerel, J. Ulrich, Structural investigation on sodium-2-keto-l-gulonate-monohydrate, Journal of Molecular Structure 1020 (2012) 121 - 126. doi:10.1016/j.molstruc.2012.03.062.

[14] T. Domschke, M. Merger, G. Grossmann, T. Faust, Method for the production of 2-keto-lgulonic acid c4-c10 alkyl esters, EP 1562965 B1 (2006).

[15] K. S. Pitzer, Activity Coeffcients in Electrolyte Solutions, 2nd Edition, CRC Press: Boca Raton, 1991.

[16] D. R. Lide, CRC Handbook of Chemistry and Physics, 77th Edition, CRC Press: Boca Raton, 1997. 